\title{
Standardization Activity on User Centric Space
}

\author{
Juyoung Park*, Sungkee Noh*, Okjo Jung* \\ *ETRI(Electronics and Telecommunications Research Institute), Rep. of Korea \\ jypark@etri.re.kr, sknoh@etri.re.kr,okjo@etri.re.kr
}

\begin{abstract}
User-Centric work Space (UCS) service provides a user with a personalized (user-centric) work space by orchestrating local and/or remote IT resources (such as file, printer, monitor, keyboard and more). With UCS service, various mobile devices such as smart phone can create personalized and secure work environment for user, because it can store and process user data in secure manner without the interference from the external environment. During the Nov. 2013 ITU-T SG13 meeting, a new standardization activity on UCS has started. With this paper, we describe the conceptual model of UCS, related standardization activity, and some of use cases to present User-Centric work Space (UCS) services.
\end{abstract}

Keywords-User Centric Space, smart work, BYOD

\section{INTRODUCTION}

According to the Gartner, the bring-your-own-device, or BYOD, phenomenon is mentioned as the "most radical shift in enterprise client computing since the introduction of the PC." BYOD is a phrase that has become widely adopted to refer to employees who bring their own computing devices - such as smartphones, laptops and PDAs - to the workplace for use and connectivity on the corporate network. BYOD makes it possible to create personalized and secure work environment for user, because it can store and process user data in secure manner without the interference from the external environment. The greatest advantage of BYOD is that the user can take its own work environment when travelling and visiting other offices. To increase portability, BYOD devices are normally small.[1]

However, this characteristic makes it difficult for user to work such as word processing. Assume that a user can make use of various office devices (such as monitor, keyboards, printers, facsimiles and more) that are available; the work environment would be more efficient. In this case, the user can connect to the available devices to make this possible. In order to do this, many issues should be covered such as peripheral device discovery, air connection, AAA, etc. However, the scope of current BYOD does not deal with these issues.

The User-Centric work Space (UCS) service provides a user with a personalized (user-centric) work space by orchestrating local and/or remote IT resources (such as file, printer, monitor, keyboard and more).

In the last ITU-T SG13 Q.1 meeting in Kampala, Uganda, we have submitted contribution ("Proposal of New Work Item on Use cases of UCS Service") to start a new work item to define use cases on UCS service.
This paper introduces the concept of "Use cases of UserCentric work Space (UCS) Service" and its standardization activity in ITU-T SG13 Q.1 group.

\section{ITU-T STANDARDIZATION ACTIVITIES[2]}

The ITU-T is one of the three sectors of the ITU (International Telecommunication Union); ITU consists of three sectors, ITU-T, ITU-R, and ITU-D. ITU-T has a number of study groups which assemble experts from around the world to develop international standards known as ITU-T Recommendations. The following Table 1 shows roles of each ITU-T study groups.

TABLE 1. ITU-T STUDY GROUPS AND THEIR ROLE

\begin{tabular}{ll} 
Study Group & \multicolumn{1}{c}{ Role } \\
SG2 & Operational aspects \\
SG3 & Economic and policy issues \\
SG5 & Environment and climate change \\
SG9 & Broadband cable and TV \\
SG11 & Protocols and test specifications \\
SG12 & Performance, QoS and QoE \\
SG13 & Future networks (\& cloud) \\
SG15 & Transport, Access and Home \\
SG16 & Multimedia \\
SG17 & Security \\
\hline
\end{tabular}

Among the study groups, SG13 focuses on the standardization activity of future networks (and cloud). Currently the number of studies under SG13 is 19 in total and each of them composes a Question. Among the 19 Questions under SG13, Question 1 works on "Service scenarios, deployment models and migration issues based on convergence services". Service scenarios developed by this group can be used by telecommunication operators while designing network environment as well as innovating business models. In either case these scenarios should be developed from the user point of view. .

According to the requirements, currently Question 1 is developing the following recommendations:

- Y.2232, NGN convergence service model and scenario using web services

- Y.2235, Converged web-browsing service scenarios in NGN

- Y.2237, Functional model and service scenarios for QoS enabled mobile VoIP service 
- Y.Sup14, ITU-T Y.2000-series - Supplementary service scenarios for fixed-mobile convergence

- Y.Sup15, ITU-T Y.2000-series - Profile-based application adaptation service using next generation networks

Also the Question 1 is developing service scenarios and deployment models related to network technologies which are in the scope of SG 13. The scopes of Question 1 include:

- Identification of service scenarios and deployment models related to new application services stemming from convergence

- Identification of service scenarios and deployment models which result from the consideration of network evolution and related technologies

- Identification of migration issues as the evolution of information and telecommunications technologies continues

- Identification as to how these service scenarios and deployment models could impact directly or indirectly other industries.

According to the aim of Question 1, it has been agreed to develop a new document on 'UCS service use cases' in ITU-T SG13 Question 1 from Nov. 2013. [3]

The concept and use case of UCS service is presented in the following clause.

\section{III.UCS SERVICE}

\section{A. Overview}

Usually smart device can create personalized and secure work environment for a user, because it can store and process user's data securely without being interfered from the external environment. The greatest advantage of this device is that the user can take its own work environment such as for checking e-mail or for reading documents, even he travels abroad, rests at home or visits his colleague's office.

Normally, to increase portability, the size of smart device tends to small. But this characteristic makes user difficult to work such as word processing.

To solve this problem we proposed a new concept called User Centric Space (UCS) service. This concept comes from the following assumption; if a user can utilize nearby office peripheral devices (such as monitor, keyboards, printers, facsimiles and more) instead of using its own user interface, the work environment for word processing would be more efficient. In order to do this, many issues should be covered such as peripheral device discovery, air connection, AAA, etc.

Also in the present work environment a user must adapt oneself into the work environment provided. For example if the user has a document to print out; although there is a brand new laser printer but he has to find someone to give him a right to use it; even though he got a right to use the laser printer, he has to find and install $\mathrm{S} / \mathrm{W}$ driver for the printer. As an one more example, a user has made a document all night long with his PC at home, the very next day he stores his document in his portable storage and goes to work; at his office, he finds the version of word processor installed in the
PC is too old and he has to reinstall a new one to continue his work.

But with UCS, the work environment can be changed drastically; instead of the user's adaption to a given work environment, the work environment can be adapted by the user. The following Figure 1 shows how the present work environment can be changed into future work environment, i.e., location-free and User-centric work environment. The left picture of the Figure 1 shows generic location-based work environment of present days, a user has to adapt himself to the given work environment; outdoor, home or office. If he does not want to bring his own laptop computer, he should store his data in the portable or network storage and then he adjust given PC to workable. In UCS service, user's device discovers available nearby peripheral devices and by fetching information of available remote resources stored in the network server, it provides user-centric work environment The right-side of Figure 1shows the location-free user-centric work environment provided by UCS service.

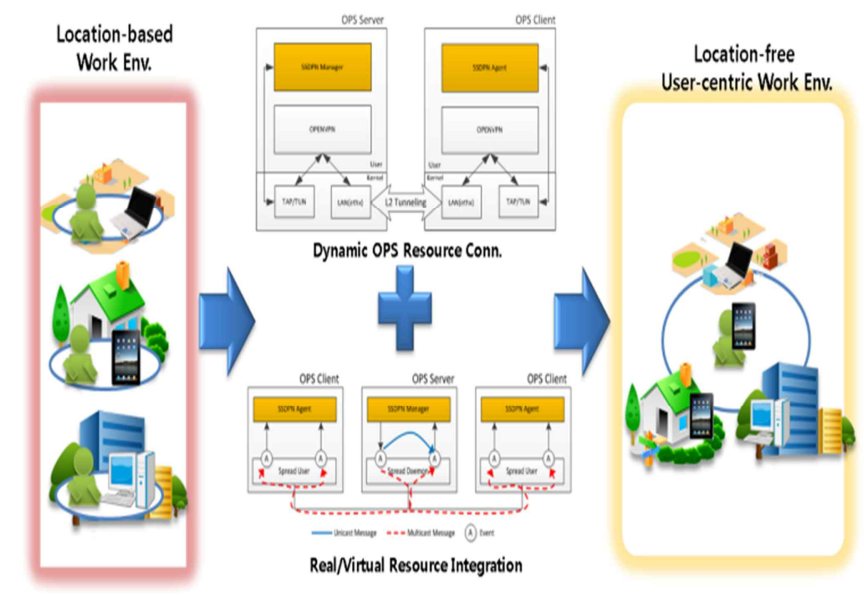

Figure 1. Location-free User-centric work environment using UCS

As a result, with UCS (User-Centric work Space) service a user can utilize nearby and/or remote IT devices just as they are directly connected to realize true personalized nomadic work environment as shown in Figure 2.

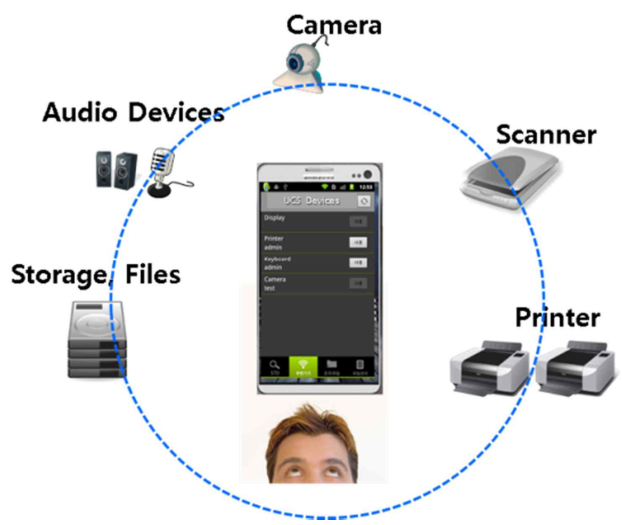

Figure 2. Concept of User-Centric work Space 


\section{B. Details}

To implement UCS service, UCS enabled agents are installed in the peripheral devices and controlled by UCS device. All the UCS services are managed by UCS service server in the network side. The followings are the control modules required by each UCS entities.

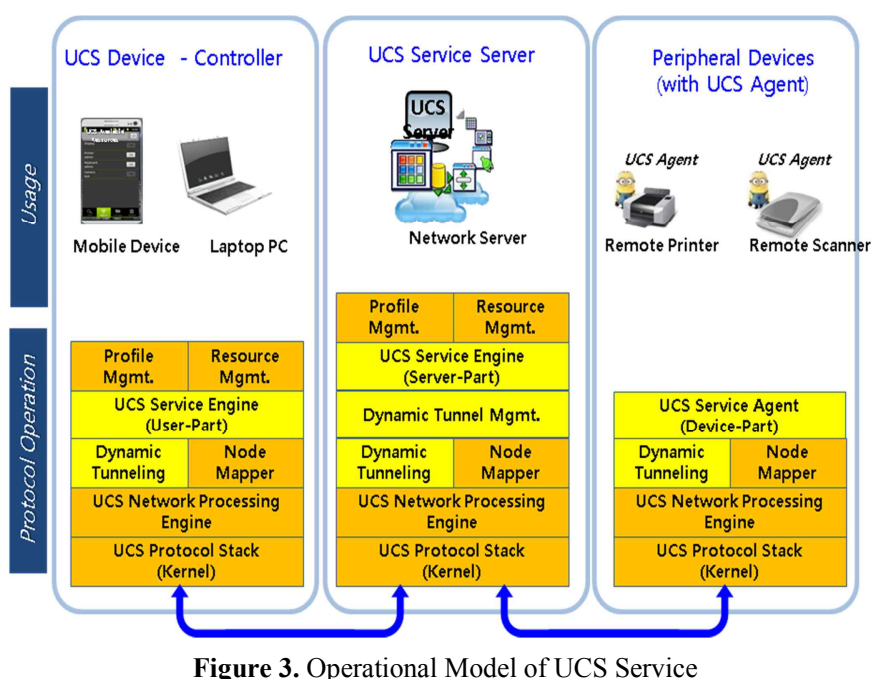

Profile management module

The device side profile management module is to manage information regarding user-oriented work environment. In which, user ID, preferred user resources are stored. The server side profile management module is to manage information regarding the construction of multiple user-oriented work environments. When there is a contention for a same resource or connect to a remote resource it manages multiple users' profiles to resolve the contention. In which, multiple user ID, preferred user resources are stored.

- Resource management module

Device side resource management module is to manage information regarding resources. In which, preferred and available user resources are stored. This module is to manage resource allocation among multiple user-oriented work environments. In which, informations of available UCS resources are stored.

- UCS service engine

It is to exchange UCS events (protocol messages) and process appropriate actions according to the event.

- Dynamic tunnelling module

It is to connect UCS entities to construct User centric network environment.

- Dynamic tunnelling management module

It is to manage dynamic tunnelling among UCS entities.

- Node mapper module

It is to connect and identify peripheral devices located inside broadcast LAN environment.
- UCS network processing engine

It is to exchanged UCS message and media.

- UCS protocol stack

It consists of legacy TCP/IP protocol stack.

The following Figure 4 shows how user interface of UCS device can be presented to users. When a user starts a UCS service, it then presents available local/remote resources to user. Depending on how UCS service is implemented, it can automatically connect available resource according to user's preference or usage history, or asks user to select wanted resources manually.

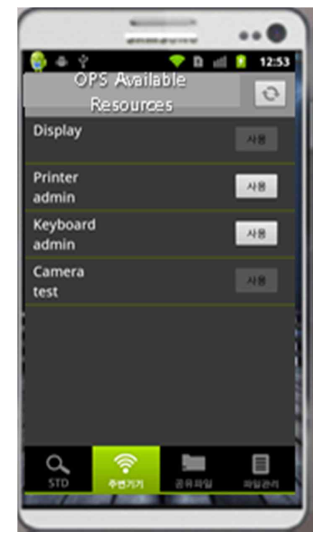

Figure 4. Conceptual UI of UCS Device

The following section continues how UCS service can be deployed in the near future.

\section{IV.SERVICE USE CASES OF UCS}

We can say that the current work environment is configured by machine. When a work environment is changed caused by user's going to business trip or going to work, he has to face a new work environment; unfamiliar desktop PC, unknown printer and so on. Therefore, the user tends to bring his computing device and data which is stored in USB memory to maintain his own work environment; however he cannot avoid of installing a new printer $\mathrm{S} / \mathrm{W}$ driver to utilize it.
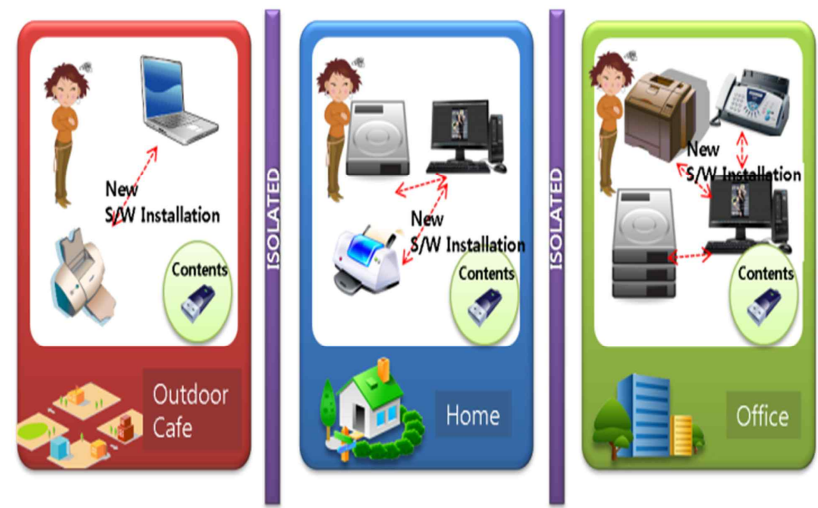

Figure 5. Service model without UCS service capability 
Figure 5 shows an example of current work environment; at every place a user visits, he has to bring his USB memory stick and adapt himself to a new work environment by searching and installing a new printer $\mathrm{S} / \mathrm{W}$ driver, installing word processing $\mathrm{S} / \mathrm{W}$ and so on.

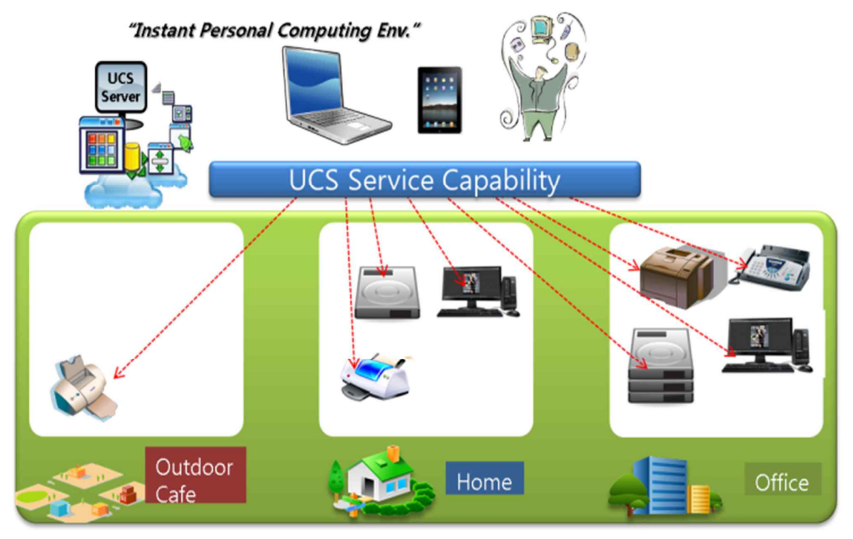

Figure 6. Service model with UCS service capability

Figure 6 shows a near future work environment with UCS capability. When a user brings a UCS enabled mobile device, the UCS dynamically connects nearby or remote IT resources such as nearby webcam, local printer or remote file systems as well as his own work environment. As a result, he need not have to install $\mathrm{S} / \mathrm{W}$ driver nor physically connect a nearby device.

A user brings his mobile device with UCS capability to a meeting room. The meeting room provides an extra wide monitor, a number of keyboards that can be utilized by any visitors. The user can connect the peripherals to his mobile device to do the final modification on meeting documents and then can share the final meeting documents projected in the extra wide monitor. In this scenario on can utilized his own work environment provided by his mobile device.

\section{Conclusion}

This paper has presented a User-Centric Space (UCS) service scheme and its standardization activity in Question 1 of ITU-T SG13. This paper has also presented a possible use case in areas of office environment. The on-going UCS implementation work is expected to be finished in two or three years the standardization works on use case will also is expected to be finalized one year before.

\section{ACKNOWLEDGMENT}

This research is supported by the ICT Standardization program of MSIP (Ministry of Science, ICT \& Future Planning).

\section{REFERENCES}

[1] Gartner's analysis on BYOD,

http://www.gartner.com/technology/topics/byod.jsp

[2] http://www.itu.int/en/ITU-T/studygroups/2013-

2016/13/Pages/default.aspx
[3] ITU-T WD, Output - text of new supplement ITU-T Y.UCS-usecase "Use cases of User-Centric work Space (UCS) service", Nov. 2013.

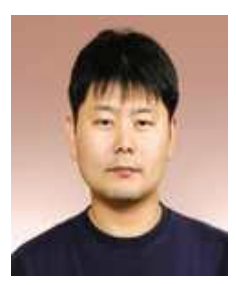

Juyoung Park He is working for ETRI from when he has received his PH.D degree in 2001 from Chungnam National University.

Thereafter, he took project editorships both in ITU-T and ISO/IEC/JTC1, and he also has developed three International Standards (IS).

His major research areas are Smartwork, Multicast, QoS protocol and architecture. He also has great concerns on mobile communication and IOT.

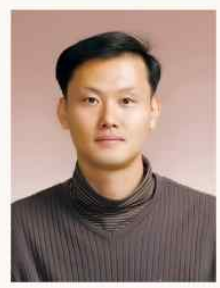

SungKee Noh He is working for ETRI from 1992. And he received his PH.D degree in 2006 from Chungnam National University. He took project for omniflow network system. Recently he is salso developing trust network gateway for secure packet transmission. His major research areas are trusted network, QoS protocol and architecture. He also has great concerns on mobile communication and mobile QoS

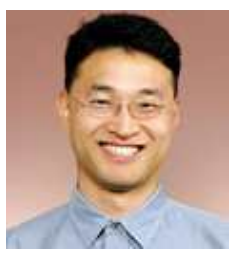

Okjo Jeong received M.S. degree in computer science from the Seoul City University in 2001.

$\mathrm{He}$ is working in ETRI as a researcher in the Standard Research Department.

Topic of interests includes video conferencing, smart work, voice over IP, voice phishing, and multicasting. 